\title{
Uterine leiomyosarcoma metastasizing to multiple sites: a rare presentation
}

\author{
Uma Handa ${ }^{1}$, Reetu Kundu ${ }^{1}$, Reeti Mehra ${ }^{2}$, Romeeta Trehan $^{3}$, Harsh Mohan $^{1}$ \\ ${ }^{1}$ Department of Pathology, Government Medical College and Hospital, Chandigarh-160030, Punjab, India \\ ${ }^{2}$ Department of Obstetrics \& Gynecology, Government Medical College and Hospital, Chandigarh-160030, Punjab, \\ India \\ ${ }^{3}$ Department of Radiotherapy, Government Medical College and Hospital, Chandigarh-160030, Punjab, India
}

Received: 13 March 2014

Accepted: 25 March 2014

\section{*Correspondence:}

Dr. Reetu Kundu,

E-mail: reetukundu@gmail.com

(C) 2014 Handa U et al. This is an open-access article distributed under the terms of the Creative Commons Attribution Non-Commercial License, which permits unrestricted non-commercial use, distribution, and reproduction in any medium, provided the original work is properly cited.

\begin{abstract}
Uterine leiomyosarcoma is a rare malignant neoplasm which has a dismal outcome especially when associated with widespread metastasis. It shows early metastasis primarily to the lungs, peritoneal cavity, bones, pelvic and paraaortic lymph nodes. We report simultaneous metastasis to the scalp, breast and soft tissue from leiomyosarcoma arising in the uterine broad ligament. Leiomyosarcoma poses a diagnostic difficulty at the metastatic site, especially when the primary site is unknown. Correct tumor typing and separating a primary from a metastatic one is important because of the different treatment modalities for both. Fine Needle Aspiration Cytology (FNAC) has emerged as an indispensable preliminary modality in investigating the metastatic disease and therefore cytomorphological recognition of the tumor presumes a great significance.
\end{abstract}

Keywords: Uterine, Leiomyosarcoma, Metastasis, Cytomorphology, FNAC

\section{INTRODUCTION}

Uterine leiomyosarcoma is rare malignant neoplasm accounting for $1-5 \%$ of uterine malignancies. ${ }^{1}$ It shows early metastasis primarily to the lungs, peritoneal cavity, bones, pelvic and para-aortic lymph nodes. ${ }^{1,2}$ Widespread metastasis indicates a dismal outcome. ${ }^{2}$

Fine Needle Aspiration Cytology (FNAC) has emerged as an indispensable preliminary modality in investigating the metastatic disease and therefore cytomorphological recognition of the tumor is of great importance. Metastases of uterine leiomyosarcoma to the breast and scalp are rare..$^{3-6}$ To the best of our knowledge, this is the first report of simultaneous metastasis to scalp, breast and soft tissue from leiomyosarcoma arising in the uterine broad ligament.

\section{CASE REPORT}

A 48-year-old multiparous female presented to the FNAC clinic with a palpable breast lump noticed a week ago. The lump was firm and mobile located in the upper outer quadrant of left breast and measured $1.5 \mathrm{~cm}$ in diameter. Mammography was not done. On further clinical examination, a tiny scalp swelling measuring $0.8 \mathrm{~cm}$ in diameter and swelling back measuring $1 \mathrm{~cm}$ in diameter were noticed.

Her past history revealed that a month ago she presented with menorrhagia to the gynecologist. Ultrasonography abdomen \& pelvis showed a large pelvic mass. Magnetic Resonance Imaging (MRI) scan showed a well-defined mass in uterine broad ligament measuring $15 \times 12.8 \mathrm{~cm}$. The mass caused inferior and left side displacement of uterus. Both the ovaries were separately visualized. The 
impression was large heterogeneous malignant mass broad ligament uterus.

Fine Needle Aspiration (FNA) was done from lump breast, swellings scalp and back using $22 \mathrm{G}$ needle attached to a $10 \mathrm{ml}$ syringe, mounted on Cameco's handle. The smears were air dried and wet fixed in 95\% alcohol for May Grünwald Giemsa (MGG) and hematoxylin and eosin (H\&E) staining, respectively. Material for cell block preparation was available in FNA from lump breast. Immunohistochemistry (IHC) for smooth muscle actin (SMA) was done on cell block.

\section{Cytologic examination}

The smears from lump breast, swellings scalp and back showed similar morphology and were cellular, showing loosely structured clusters and scattered tumor cells (Figure 1a). Cells were spindle shaped anisokaryotic with a syncytial appearance and ill-defined borders (Figure 1b). The nuclei were oval to spindly, cigar shaped with blunt ends with malignant chromatin pattern. The cytoplasm was moderate to abundant with perinuclear vacuolations. Few mitoses including atypical ones and occasional multinucleated giant cells were noted. IHC for SMA was positive.

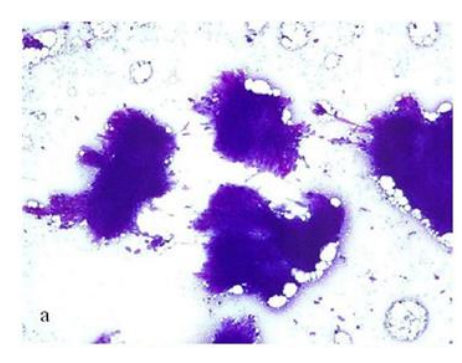

Figure 1a: Fine-needle aspirate from lump breast: cellular smear with loosely cohesive sheets and clusters of tumor cells (MGG, x100).

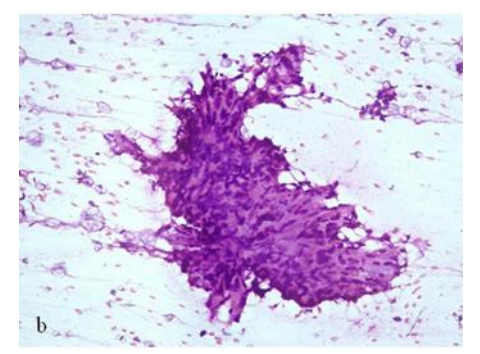

Figure 1b: Fine-needle aspirate from lump breast: Spindle shaped anisokaryotic tumor cells arranged in parallel fashion with a syncytial appearance and oval to spindly nuclei having blunt ends (H\&E, x200).

\section{Histopathologic examination}

A panhysterectomy with removal of uterine broad ligament mass and infracolic omentectomy done earlier showed the mass to be lobulated measuring $14 \times 9 \times 5$ $\mathrm{cm}$. It had a soft fleshy consistency and a variegated cut surface, grey yellow to pink with foci of haemorrhage and necrosis. On histopathologic examination the tumor was composed of interlacing fascicles of pleomorphic spindle shaped cells with 10 mitoses per high power field (Figure 1c). Large areas of necrosis were seen. A panel of immunostains was done. The tumor cells were positive for SMA (Figure 1d), desmin and vimentin. Proliferation marker Ki-67 was diffusely and strongly positive. Cytokeratin (CK), S-100, CD 34 and CD 117 were negative. The uterine corpus, cervix, bilateral adnexa and excised omentum were free of any tumor involvement. The histopathologic diagnosis was leiomyosarcomauterine broad ligament.

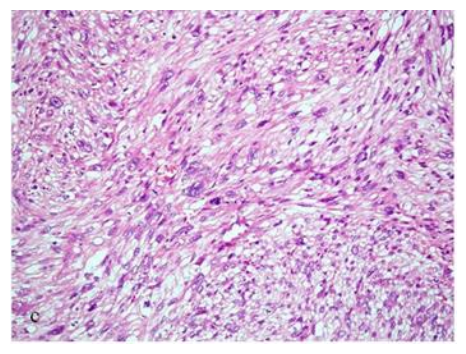

Figure 1c: Histopathology section of uterine broad ligament mass showing interlacing fascicles of pleomorphic spindle shaped tumor cells with brisk mitotic activity (H\&E, x200).

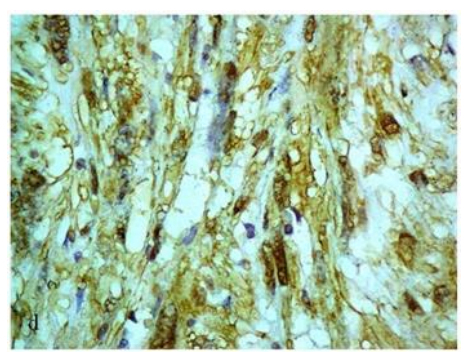

Figure 1d: Tumor cells showing intense cytoplasmic immunopositivity for SMA (IHC, x600).

The diagnosis rendered on FNAC was metastatic leiomyosarcoma in a known case. Subsequently the patient was put on chemotherapeutic regimen comprising of adriamycin and ifosphamide. Clinically there was regression of lesions and positron emission tomography scan showed partial response.

\section{DISCUSSION}

Uterine leiomyosarcoma has a strong predilection for widespread distant metastasis, in keeping with its aggressiveness and propensity for hematogenous spread. Distant metastases in uterine leiomyosarcoma usually occur long after hysterectomy. ${ }^{3}$ Metastases of uterine leiomyosarcoma to the breast and scalp are rare and only five cases of each have been reported in the medical literature. ${ }^{3-6}$ The first case metastatic to the breast was 
reported way back in 2003 while that with scalp being the metastatic site was reported much earlier in $1917 .^{3,6}$

The cytomorphologic features described in leiomyosarcoma and well visualised in our case are cellular smears composed of tissue fragments, clusters and fascicles of cohesive tumor cells. ${ }^{7-10}$ Elongated nuclei with blunt ends and a vague " $\mathrm{C}$ " shape containing small amount of wispy cytoplasm with bipolar cytoplasmic processes may be appreciated. Stripped atypical nuclei may be seen in the background. Mitotic figures and necrosis when present indicate malignancy. ${ }^{10}$ Leiomyosarcoma can have varied cell morphologies. Intranuclear cytoplasmic invaginations have been reported although these were absent in the present case. Signet ring cell appearance in occasional tumor cells may be seen. ${ }^{9}$ At times multiple osteoclast-like giant cells are present.

The cytologic differentiation of leiomyosarcoma at metastatic sites is a diagnostic challenge. The differential diagnoses include other spindle cell sarcomas like fibrosarcoma, neurogenic tumors and Malignant Fibrous Histiocytoma (MFH). ${ }^{9,11,12}$ When seen in breast the list becomes exhaustive and also includes phyllodes tumor, sarcomatoid carcinoma, metaplastic carcinoma, myoepithelial carcinoma and primary sarcomas of the breast.

Malignant peripheral nerve sheath tumor has pointed nuclei and slender bipolar cytoplasmic tails. Presence of multinucleated tumor giant cells indicates MFH. Myoepithelial carcinoma shows clusters of small spindled or epithelioid tumor cells with some nuclear atypia. Highgrade phyllodes tumor is a dimorphic tumor with sheets of ductal cells in addition to the spindle cells. Further, leiomyosarcomas are positive for SMA, desmin, vimentin and negative for CK and S-100. ${ }^{9}$ Neurogenic tumors and myoepithelial carcinomas are positive for $\mathrm{S}-100$. The stromal component of metaplastic carcinoma is positive for CK, vimentin and S-100.

To conclude, leiomyosarcoma with widespread dissemination is rare and poses a diagnostic difficulty at the metastatic site, especially when the primary site of tumor is unknown. In such cases, a careful clinical examination and thorough work-up for exclusion of other organ involvement are mandatory. The diagnosis was easier in the present case as the primary diagnosis was known. Correct tumor typing and separating a primary from a metastatic one is important as there are different treatment modalities for both.

Funding: No funding sources Conflict of interest: None declared Ethical approval: Not required

\section{REFERENCES}

1. Yamada S, Yamada SM, Nakaguchi H, Murakami M, Hoya K, Matsuno A. A case of multiple brain metastases of uterine leiomyosarcoma with a literature review. Surg Oncol. 2011;20(4):e127-31.

2. Lusby K, Savannah KB, Demicco EG, Zhang Y, Ghadimi MPH, Young ED et al. Uterine leiomyosarcoma management, outcome, and associated molecular biomarkers: a single institution's experience. Ann Surg Oncol. 2013;20(7):2364-72.

3. Sibartie S, Larkin JO, Lee G, Fitzgibbon J, O'Reilly $\mathrm{S}$, Richardson D. Metastatic uterine leiomyosarcoma presenting as a breast lump. Ir J Med Sci. 2011;180(4):889-91.

4. Pappa L, Zagorianakou N, Kitsiou E, Sintou-Mantela E, Bafa M, Malamnou-Mitsi V. Breast metastasis from uterine leiomyosarcoma diagnosed by fine needle aspiration: a case report. Acta Cytol. 2008;52(4):485-9.

5. Hsiao HH, Liu YC, Hou MF, Lin SF. Uterine leiomyosarcoma metastasis to the breast. Eur J Gynaecol Oncol. 2008;29(2):191-2.

6. Corcoran S, Hogan AM, Nemeth T, Bennani F, Sullivan FJ, Khan $\mathrm{W}$ et al. Isolated cutaneous metastasis of uterine leiomyosarcoma: case report and review of literature. Diagn Pathol. 2012;18(7):85

7. Karabulut Z, Akkaya H, Moray G. Primary leiomyosarcoma of the breast: a case report. J Breast Cancer. 2012;15(1):124-7.

8. Shukla R, Pooja B, Radhika S, Nijhawan R, Rajwanshi A. Fine-needle aspiration cytology of extramammary neoplasms metastatic to the breast. Diagn Cytopathol. 2005;32(4):193-7.

9. Jun Wei X, Hiotis K, Garcia R, Hummel-Levine P. Leiomyosarcoma of the breast: a difficult diagnosis on fine-needle aspiration biopsy. Diagn Cytopathol. 2003;29(3):172-8.

10. Omeroglu G, Erssahin C, Potkul RK, Booth CN. FNA diagnosis of retroperitoneal leiomyosarcoma metastasizing to the breast. Diagn Cytopathol. 2007;35(8):508-11.

11. Rodríguez-Gil Y, Pérez-Barrios A, Alberti-Masgrau N, Garzón A, de Agustín P. Fine- needle aspiration cytology diagnosis of metastatic non haematological neoplasms of the breast: a series of seven cases. Diagn Cytopathol. 2012;40(4):297-304.

12. Gupta RK. Needle aspiration cytology and immunohistologic findings in a case of leiomyosarcoma of the breast. Diagn Cytopathol. 2007;35(4):254-6.

DOI: $10.5455 / 2320-1770$. ijrcog20140635

Cite this article as: Handa U, Kundu R, Mehra R, Trehan R, Mohan H. Uterine leiomyosarcoma metastasizing to multiple sites: a rare presentation. Int J Reprod Contracept Obstet Gynecol 2014;3:453-5. 